

\title{
A Dynamically Reconfigurable RF NoC for Many-Core
}

Alexandre Brière, Eren Unlu, Julien Denoulet, Andrea Pinna, Bertrand

Granado, Francois Pêcheux, Yves Louët, Christophe Moy

\section{To cite this version:}

Alexandre Brière, Eren Unlu, Julien Denoulet, Andrea Pinna, Bertrand Granado, et al.. A Dynamically Reconfigurable RF NoC for Many-Core. ACM Great Lakes Symposium on VLSI, May 2015, Pittsburgh, United States. pp.139-144, 10.1145/2742060.2742082 . hal-01166859

\section{HAL Id: hal-01166859 \\ https://hal.science/hal-01166859}

Submitted on 23 Jun 2015

HAL is a multi-disciplinary open access archive for the deposit and dissemination of scientific research documents, whether they are published or not. The documents may come from teaching and research institutions in France or abroad, or from public or private research centers.
L'archive ouverte pluridisciplinaire HAL, est destinée au dépôt et à la diffusion de documents scientifiques de niveau recherche, publiés ou non, émanant des établissements d'enseignement et de recherche français ou étrangers, des laboratoires publics ou privés. 


\title{
A Dynamically Reconfigurable RF NoC for Many-Core
}

\author{
Alexandre Brière ${ }^{1}$, Eren Unlu ${ }^{2}$, Julien Denoulet ${ }^{1}$, Andrea Pinna ${ }^{1}$, \\ Bertrand Granado ${ }^{1}$, Francois Pêcheux ${ }^{1}$, Yves Louët ${ }^{2}$, Christophe Moy ${ }^{2}$ \\ ${ }^{1}$ Sorbonne Universités, UPMC Univ Paris 06, UMR 7606, LIP6, F-75005 Paris, France \\ $<$ firsname.lastname>@lip6.fr \\ ${ }^{2}$ Supélec - IETR, F-35576 Cesson-Sevigne Cedex, France \\ $<$ firsname.lastname>@supelec.fr
}

\begin{abstract}
With the growing number of cores on chips, conventional electrical interconnects reach scalability limits, leading the way for alternatives like Radio Frequency (RF), optical and 3D. Due to the variability of applications, communication needs change over time and across regions of the chip. To address these issues, a dynamically reconfigurable Network on Chip (NoC) is proposed. It uses RF and Orthogonal Frequency Division Multiple Access (OFDMA) to create communication channels whose allocation allows dynamic reconfiguration. We describe the NoC architecture and the distributed mechanism of dynamic allocation. We study the feasibility of the NoC based on state of the art components and analyze its performances. Static analysis shows that, for point to point communications, its latency is comparable with a 256-node electrical mesh and becomes lower for wider networks. A major feature of this architecture is its broadcast capacity. The RF NoC becomes faster with 32 nodes, achieving a $\times 3$ speedup with 1024 . Under realistic traffic models, its dynamic reconfigurability provides up to $\times 6$ lower latency while ensuring fairness.
\end{abstract}

\section{Categories and Subject Descriptors}

C.1.2 [Processor Architectures]: Multiple Data Stream Architectures (Multiprocessors)-Interconnection architectures

\section{Keywords}

Many-Core; NoC; RF; Dynamic; Reconfigurable;

\section{INTRODUCTION}

With the emergence of Chip Multi Processor (CMP), Network on Chip (NoC) have been introduced to make the different processing elements soundly communicate together. First generation NoCs based on electrical interconnects have been studied in the past decade. Their bandwidth can be considered as infinite, but they show limitations in terms of

Permission to make digital or hard copies of all or part of this work for personal or classroom use is granted without fee provided that copies are not made or distributed for profit or commercial advantage and that copies bear this notice and the full citation on the first page. Copyrights for components of this work owned by others than ACM must be honored. Abstracting with credit is permitted. To copy otherwise, or republish, to post on servers or to redistribute to lists, requires prior specific permission and/or a fee. Request permissions from Permissions@ acm.org. GLSVLSI'15, May 20-22, 2015, Pittsburgh, PA, USA.

Copyright (C) 2015 ACM 978-1-4503-3474-7/15/05 ...\$15.00.

http://dx.doi.org/10.1145/2742060.2742082. latency, when the number of cores increases. To overcome these limitations, alternatives such as $3 \mathrm{D}$, optical and $R a$ dio Frequency (RF) are now explored. In this paper, we present a RF NoC with dynamic allocation of communication resources. It takes advantage of $\mathrm{RF}$ properties to optimize bandwidth utilization and reduce latency. Section 2 presents an overview of related work. Section 3 describes the RF NoC architecture. Section 4 details the dynamic allocation algorithm. Section 5 presents experimental setups and results, and section 6 concludes the paper.

\section{RELATED WORK}

\subsection{Technology trends}

Following recommendations of the ITRS [8], new technologies are explored to overcome limitations of electrical connections. As chips integrate more and more components, distances, and therefore latency, increase. Pioneering contributions try to reduce distances with $3 \mathrm{D}[12,15]$ or reduce traveling time with optic $[13,19]$ and $\operatorname{RF}[3,2]$.

\subsubsection{D NoC}

$3 \mathrm{D}$ chips are based on stacked layers. In addition to planar connections, each layer has vertical connections with other layers. Some approaches mix 3D with optic [23] or RF [11] by using dedicated layers. 3D offers many advantages, but also adds significant thermal constraints.

\subsubsection{Optical NoC}

Optical is used to provide shortcuts for long distance communications. The use of optic, like other new technologies, not only allows a gain in bandwidth and latency, but also other architectural innovations such as new cache management policies [10]. A major drawback of optical NoC is the need of an external light source.

\subsubsection{RF NoC}

Similar to the optic solution, RF waves travels at a speed close to the speed of light. But, unlike optical solutions, RF directly benefits from full compatibility with CMOS technology. RF waves can be transmitted through a waveguide [2] or an antenna [4]. Solutions using antennas have greater flexibility, but they also increase consumption compared to waveguides, and suffer from lesser immunity to interference.

\subsection{Communication Resources Allocation}

As on-chip traffic is highly heterogeneous in the spatial and temporal domain, available bandwidth is generally un- 


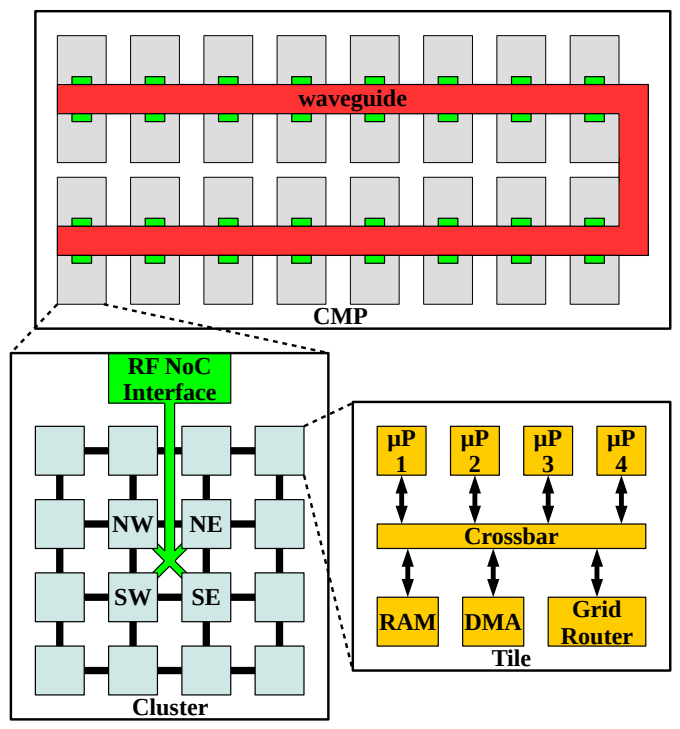

Figure 1: CMP hierarchical RF NoC architecture

derused and the design over-dimensioned in static interconnects. In optical or RF solutions, bandwidth is divided into dedicated channels that allow simultaneous transmissions. In a Single Write Multiple Read (SWMR) scheme, each communication channel is allocated to a specific node $N$ so every other node can receive data sent by $N$ [10]. In contrast, Multiple Write Single Read (MWSR) allocates each communication channels to a specific node $N$ so it can receive information from other nodes [19]. MWSR has one drawback: it requires an arbitration mechanism in the receiving devices to avoid collision, as multiple nodes may simultaneously transmit data to the same destination node. SWMR supports broadcasting, while MWSR has to copy the broadcast message to each dedicated channel nodes. In terms of energy, all nodes in a SWMR scheme listen to all communication channels in the system (i.e. whole bandwidth). In that case, a node only requires small specific hardware for its pre-dedicated bandwidth. It is the opposite for MWSR: a node only has to listen to its pre-dedicated channel and just needs dedicated hardware to emit over the whole bandwidth. In Multiple Write Multiple Read (MWMR), each node can be assigned multiple channels to emit a message, and respectively each node can simultaneously receive data from different channels and nodes [21]. MWMR is the most energy consuming, as it requires arbitration for both emission and reception. It is also by far the most flexible solution and allows more advanced reconfiguration mechanisms.

\section{PROPOSED CMP ARCHITECTURE}

This section presents the architecture of the hierarchical RF NoC based CMP. As 3D adds significant thermal constraints and optical needs an external light source, RF has been chosen. MWMR is chosen for its flexibility and broadcast capacity. The propagation time in silicon is very short at the scale of a chip (about $0.5 \mathrm{~ns}$ for $100 \mathrm{~mm}$ ), and can be considered almost constant for any source-destination combination. Moreover, the time needed to emit and receive a RF message is also constant. So, the RF NoC allows to transmit data in constant time, unlike router-based NoC, for which transmission duration varies linearly with the distance between source and destination. However, this dura- tion is still higher than a router-based NoC for short distance communications. So RF must be used only when a certain distance threshold is reached, involving the use of a hierarchical architecture where the RF NoC is the top level communication medium.

\subsection{CMP architecture}

Figure 1 shows the global architecture of the CMP, with three levels of hierarchy: tiles, clusters and CMP. Each hierarchical level is associated with a specific interconnect: respectively wired crossbar, wired grid and RF global interconnect. The tile is the lowest hierarchical level. It contains a local RAM, a DMA and processors, all connected to the crossbar. The local crossbar is connected to a grid router to access the second level (the wired grid). A tile is connected by its router to the four nearest tiles, forming a cluster of $M \times M$ tiles. As the increase of the latency limits the size of a cluster, RF NoC is used as a third level interconnect when its latency becomes lower than the grid latency.

\subsection{RF NoC architecture}

To allow inter cluster communications, each cluster includes a RF NoC interface connected on one side to the four central tiles as illustrated in Figure 1, and on the other side to the waveguide. The RF NoC Interface, pictured in Figure 2, makes use of Orthogonal Frequency Division Multiplexing (OFDM). In OFDM, the bandwidth is split into several orthogonal narrow band channels, providing high spectral efficiency, manageable equalization process and robustness. The RF NoC use specifically a medium access scheme based on OFDM called Orthogonal Frequency Division Multiple Access (OFDMA). It adds the possibility for multiple senders to simultaneously use the same bandwidth while using only one transceiver per sender.

Data coming from routers of the four central tiles are stored into corresponding FIFOs $\mathbf{1}$. The arbiter $\mathbf{2}$ selects a packet from one of the FIFOs $\mathbf{1}$, converts it into specific RF NoC flow units (flits) and writes it in the FIFO 3 which possesses all the monitoring mechanisms to evaluate the transmit requirements of the cluster. The flit is then serialized 4 into groups of bits according to the chosen modulation (BPSK, QPSK or 16-QAM), and finally creates the appropriate constellation symbol. The demux 5 parallelizes the modulator output to feed the IFFT inputs 6 , according to the channels/subcarriers allocated to the cluster. OFDM symbols generated by the IFFT $\mathbf{6}$ feeds the RF Tx and its DAC 7 and are sent to the waveguide. To transform $O F D M$ in $O F D M A$, each cluster is allocated a certain number of subcarriers at every OFDM symbol to transmit its data and perform zero-padding to the rest of subcarriers. This way, the transmitter allows other nodes to send their data on the same OFDM symbol without interference. The choice of subcarriers is indicated by the RF Controller 7 to the demux $\mathbf{5}$ by the control signal (C). The other way to tune communication resources is to change the modulation. The choice of the modulation is also performed by the RF Controller according to its allocation algorithm presented in the next section and provided to the modulator by the control signal (B). Data reception follows the opposite path in all clusters. As each cluster decodes the entire OFDM symbol, data circulating on the $\mathrm{RF}$ NoC are visible by all clusters in the CMP. Thus, broadcast is an intrinsic feature of OFDMA. To perform the dynamic bandwidth allocation, 




Figure 2: RF NoC Interface

the RF controller 7 evaluates the bandwidth requirements of its own cluster (A) and the needs of other clusters (F).

\section{DYNAMIC BANDWIDTH ALGORITHM}

To reach maximum bandwidth occupation, the OFDMA reconfigurability potential must be fully exploited. To do so, efficient algorithms based on instantaneous traffic demands are required to allocate subcarriers among clusters. The most significant metrics for these algorithms are latency and fairness. This issue is clearly linked to network theory and efficient solutions rely on state-of-the-art OFDMA schedulers, which optimize these metrics. Little's Law states that to minimize average latency, a scheduler has to maximize the use of bandwidth. Among schedulers for conventional OFDMA networks, we choose Queue Proportional Scheduler (QPS) as the best guideline to design our allocation algorithm. QPS is a throughput optimal scheduler and is proven to provide fairness and small instantaneous latency [16]. The scheduler arbitrates $N$ subcarriers to $K$ nodes, proportionally to their instantaneous queue lengths and allows to allocate more subcarriers to nodes that communicate the most.

A distributed bandwidth allocation scheme is used. In the block 3 of Figure 2, clusters compute their Queue State Information (QSI) and transmit them simultaneously with payload data to other clusters, thanks to OFDMA intrinsic broadcast feature. When each cluster has collected all other cluster's QSI (F) in Figure 2), all RF Controller simultaneously execute the bandwidth allocation algorithm. As each $\mathrm{RF}$ Controller processes identical data with the same algorithm, they all compute locally the same allocation solution, thus avoiding bandwidth conflict. Transmitting QSI simultaneously with payload and using a distributed algorithm allow to avoid latency and extra communication overhead.

QSI is computed and transmitted every $\tau$ symbol, $\tau$ being a trade-off parameter. On the one hand, a small value of $\tau$ allows optimal allocation but the algorithm is executed more often and QSI transmission more frequent, consuming more bandwidth. On the other hand, larger values of $\tau$ result in

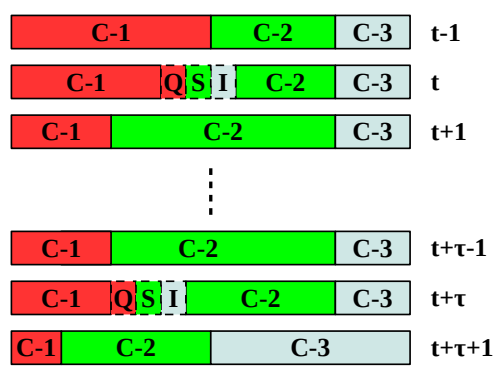

Figure 3: EQPS algorithm with rotating QSI channels on frequency and time

more available bandwidth, but outdated QSIs decrease the algorithm performance.

Expected Queue Proportional Scheduler (EQPS) algorithm is an extension of the original QPS algorithm. It provide the expected future QSI values of clusters. At first each cluster calculates its Deterministic Queue State Information (DQSI) which represents the minimum service demand. DQSI is the difference between the last received QSI and the bandwidth allocated during the last $\tau$ symbols. It is calculated using equation (1). With $Q_{i}^{t}$, the QSI of cluster $i$ received on symbol $t$ and $S_{i}^{t}$, the number of allocated subcarriers from symbol $t$ to symbol $t+\tau$. Once DQSI is obtained, it is possible to take into account the incoming data to a cluster's transmission queue during the next period of $\tau$ symbols. For this purpose, we use Exponentially Weighted Average Filter (EWMA). It estimates the next sample by weighting recent observation and previously computed average using equation (2). With $A_{i}^{t}$, the amount of bits arrived during the last $\tau$ symbols, and $\alpha$, a scalar that weighs the effect of historical data compared to instantaneous sample, which is generally between 0.9 and 0.99 [22].

$$
\dot{Q}_{i}^{t+\tau}=\min \left(0, Q_{i}^{t}-S_{i}^{t}\right)(1) \quad \hat{A}_{i}^{t+\tau}=(1-\alpha) A_{i}^{t}+\alpha \hat{A}_{i}^{t}
$$

Merging the average arrival, the last QSI value and the current bandwidth allocation, the RF Controllers use equation (3) to calculate $\hat{Q}_{i}^{t}$, the estimated QSI for cluster $i$. Then, using equation (4), they compute $S_{i}^{t+1}$, the allocated number of subcarriers to cluster $i$ on symbol $t+\tau$. With $N$, the number of subcarriers, and $K$ the number of clusters. The result is rounded to the nearest larger integer to guarantee that every non-empty queue gets at least one subcarrier resource, which ensures fairness. Note that, at the end of computation, the total number of allocated subcarriers may exceed $N$. So extra subcarriers are removed from the nodes with the higher values of $S_{i}^{t+1}$.

$$
\hat{Q}_{i}^{t}=\dot{Q}_{i}^{t}+\hat{A}_{i}^{t} \quad(3) \quad S_{i}^{t+1}=\left\lceil N \frac{\hat{Q}_{i}^{t}}{\sum_{j=1}^{K} \hat{Q}_{j}^{t}}\right\rceil
$$

To avoid using the same subcarriers for QSI transmission every $\tau$ symbols, as it would constantly induce allocation penalties in some clusters, a rotating QSI channel is used during every allocation frame. It maps the QSI channels to different adjacent blocks of subcarriers, by moving the interval one by one. This procedure is illustrated in Figure 3.

\section{EXPERIMENTAL SETUP AND RESULT}

In this section, we assume a realistic bandwidth of $20 \mathrm{GHz}$ for RF and a CMP with 32 clusters and 4 cores per tile. 


\begin{tabular}{|l||r|r|r|r|}
\hline \multicolumn{5}{|c|}{ CONFIGURATION } \\
\hline \# cores & 512 & 1024 & 2048 & 4096 \\
\hline \# tiles & 128 & 256 & 512 & 1024 \\
\hline \# clusters & 32 & 32 & 32 & 32 \\
\hline \multicolumn{5}{|c|}{ AREA } \\
\hline IFFT/FFT [18] & 20,27 & 20,27 & 20,27 & 20,27 \\
\hline Tx/Rx [9] & 1,23 & 1,23 & 1,23 & 1,23 \\
\hline DAC/ADC [5] & 7,36 & 7,36 & 7,36 & 7,36 \\
\hline Wageguide [7] & 4,35 & 6,16 & 8,71 & 12,31 \\
\hline RF NoC & $\mathbf{3 3 , 2 1}$ & $\mathbf{3 5 , 0 2}$ & $\mathbf{3 7 , 5 7}$ & $\mathbf{4 1 , 1 8}$ \\
\hline Cores & 105,32 & 210,64 & 421,27 & 842,55 \\
\hline RAM [6] & 4,50 & 8,99 & 17,99 & 35,98 \\
\hline CMP & $\mathbf{1 4 3 , 0 3}$ & $\mathbf{2 5 4 , 6 5}$ & $\mathbf{4 7 6 , 8 3}$ & $\mathbf{9 1 9 , 7 0}$ \\
\hline RF NoC part & $\mathbf{2 3 , 2 2 \%}$ & $\mathbf{1 3 , 7 5 \%}$ & $\mathbf{7 , 8 8 \%}$ & $\mathbf{4 , 4 8 \%}$ \\
\hline \multicolumn{5}{|c|}{ POWER } \\
\hline IFFT/FFT $[18]$ & 4320 & 4320 & 4320 & 4320 \\
\hline Tx/Rx $[9]$ & 400 & 400 & 400 & 400 \\
\hline DAC/ADC $[5]$ & 5184 & 5184 & 5184 & 5184 \\
\hline RF NoC & $\mathbf{9 ~ 9 0 4}$ & $\mathbf{9 ~ 9 0 4}$ & $\mathbf{9 ~ 9 0 4}$ & $\mathbf{9 ~ 9 0 4}$ \\
\hline Cores & 19896 & 39793 & 79586 & 159172 \\
\hline RAM [6] & 1446 & 2891 & 5782 & 11564 \\
\hline CMP & $\mathbf{3 1 ~ 2 4 6}$ & $\mathbf{5 2 ~ 5 8 8}$ & $\mathbf{9 5} \mathbf{2 7 2}$ & $\mathbf{1 8 0 ~ 6 4 0}$ \\
\hline RF NoC part & $\mathbf{3 1 . 7 0 \%}$ & $\mathbf{1 8 , 8 3 \%}$ & $\mathbf{1 0 , 4 0 \%}$ & $\mathbf{5 , 4 8 \%}$ \\
\hline
\end{tabular}

Table 1: Chip area $\left(\mathrm{mm}^{2}\right)$ and power $(\mathrm{mW})$

\subsection{Surface and power estimation}

In order to evaluate the feasibility of a CMP using our RF NoC, we perform a basic estimation of surface and power. We compare these characteristics with those of an existing CMP, the Intel Core i7-5960X.

\subsubsection{Experimental setup}

Surface and power are calculated on the basis of state of the art components, assuming a $22 \mathrm{~nm}$ target technology as used for the i7-5960X. For digital components, equation (5) is used to get $S_{N}$, the normalized surface in the target technology $N$, depending on the surface $S_{Q}$ in the original technology $Q$. According to ITRS [8], when the size of transistors decreases by a 0.7 factor, the power consumption decreases by a 0.65 factor. Equation (6) gives $W_{N}$, the normalized power consumption in technology $N$, based on the power consumption $W_{Q}$ in the original technology $Q$. The scaling of analog components is not as simple, since it is necessary to take into account other factors. So we directly used the surface and power of state of the art components.

$$
S_{N}=S_{Q} \times(N / Q)^{2}(5) \quad W_{N}=W_{Q} \times 0.65^{\log _{0.7}(N / Q)}
$$

\subsubsection{Result}

Table 1 presents surface and power of the components, showing that the RF NoC area is mainly due to FFT/IFFT, $\mathrm{ADC} / \mathrm{DAC}$ and the waveguide. The rest of the surface is mainly occupied by cores and their caches (ARM CortexA5). Compared to the i7-5960X, whose surface is $355 \mathrm{~mm}^{2}$, the first three cases have a realistic area. The last one seems less realistic with current technology but should be achievable in the future. Power consumption and surface do not vary in the same way. This is due to the increasing length of the waveguide. The surface calculation uses the true length of the waveguide whereas the power calculation uses the average length of the different cases. Table 1 shows that the power consumption of the $\mathrm{RF}$ NoC is mainly due to FFT/IFFT and ADC/DAC. Compared to the i7-5960X, whose power consumption is $140 \mathrm{~W}$, all cases are realistic, demonstrating the feasibility of the $\mathrm{RF}$ NoC from this point of view. The part of the RF NoC in the global surface and

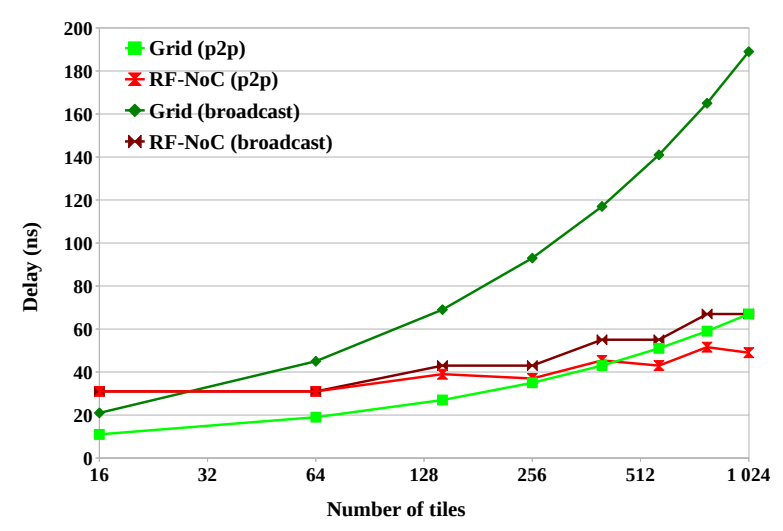

Figure 4: Average latency for P2P flit transfer

power decreases as the number of cores increase, making the $\mathrm{RF}$ NoC more and more interesting.

\subsection{Intrinsic characteristic evaluation}

\subsubsection{Experimental setup}

The $\mathrm{RF}$ NoC is compared with a router based electrical NoC using analytical models. Performance are evaluated in the case of a random traffic without contention. We assume cores running at $1 \mathrm{GHz}$ and a bandwidth divided into 512 subcarriers. A grid router has a latency $l_{R G}$ of 3 cycles, one for reading data, one for selecting the output port and one for writing data to the chosen port. Equation (7) gives $D_{C M P}$, the average number of crossed routers to go from one tile to another, including the original tile router. We considered a CMP with a grid of $N \times N$ tiles. We get $L_{\text {grid }}$ the average latency of a grid with equation (8).

$$
D_{C M P}=\frac{2}{3} N+1 \quad \text { (7) } \quad L_{\text {grid }}=D_{C M P} \times l_{R G}
$$

The latency of the RF NoC $l_{R F}$ is 25 cycles, which corresponds to the OFDM symbol duration. We also take into account the average number of cycles to go from source to the transmitting $\mathrm{RF}$ NoC Interface and from the receiving $\mathrm{RF}$ NoC Interface to destination. For a CMP containing clusters of $M \times M$ tiles, the average distance $D_{\text {cluster }}$ between tiles and $\mathrm{RF}$ router is given by equation (9) if $M$ is even, and equation (10) if $M$ is odd. Equation (11) gives $L_{N o C_{-} R F}$ the average latency of the RF NoC.

$$
\begin{gathered}
D_{\text {cluster }}=\frac{M}{2} \text { (9) } D_{\text {cluster }}=\frac{M^{2}+2 M-1}{2 M} \\
L_{N o C_{-} R F}=l_{R G} \times D_{\text {cluster }}+l_{R F}
\end{gathered}
$$

We can compare the average duration of a flit reception with the $\mathrm{RF}$ NoC and with a grid connecting all the tiles of the CMP. We set the number of clusters to 32 and we modify the size of CMP by varying the number of tiles per cluster.

\subsubsection{Result}

Access to the RF NoC is shared by the four central tiles in the first case, whereas it is in the single central tile in the second case. This different location of the RF router explains the staircase shape of the RF NoC latency curves shown in Figure 4. For point-to-point (P2P) communications, the RF NoC becomes faster for CMPs of at least 512 tiles.

To perform a broadcast, data must cross a maximum of $2 N-1$ routers for a grid and a maximum of $2 M$ routers plus the waveguide for the RF NoC, where $N$ is the CMP 


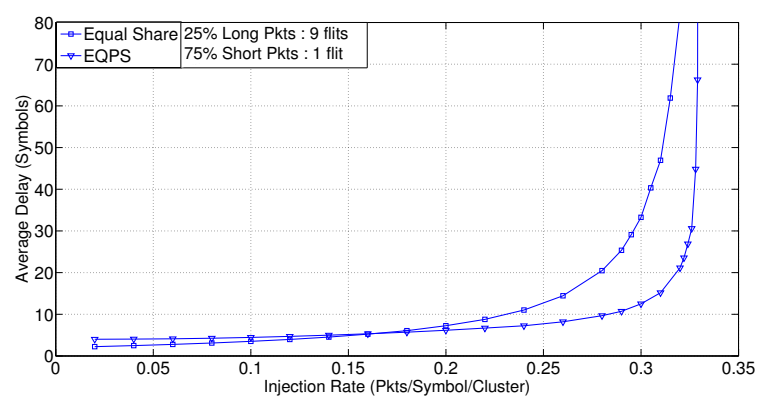

Figure 5: Average latency under Poisson traffic

width while $M$ is the cluster width. Figure 4 shows that the $\mathrm{RF}$ NoC is faster for CMPs of at least 32 tiles.

\subsection{Evaluation of dynamic allocation}

\subsubsection{Experimental setup}

Note that in this experiment, QPSK is the only used modulation order. Based on experiments, we decide to compute QSI every 8 symbols. It provides the necessary amount of computation time for the RF Controller to perform subcarriers allocation while only $3.125 \%$ of bandwidth is used for QSI transmission. Assuming that the shortest packet is one flit, incoming packets are split into 64 bits flits. We divide the 1024 subcarriers into 256 groups of 4 subcarriers modulated with QPSK during 8 symbols, thus providing 64 bits of service during every allocation frame and ensuring that each non-idle node gets a chance to transmit at least one flit. QSI values are 8-bit long, representing a queue of 0 to 255 of awaiting flits. That way, we only need 4 subcarriers to modulate a cluster's QSI on one symbol. So, we use 128 subcarriers during one symbol to transmit all the QSIs.

Messages are either small packets (read requests, acknowledgements, etc.), or long packets (write requests, read responses followed by cache lines). According to [14], we set the proportion of short packets ( 1 flit - 64 bits) and long packets (9 flits - 576 bits) to $75 \%$ and $25 \%$. It is assumed that cache lines are 64 bytes, thus cache coherence packets contain 8 payload flits and 1 overhead flit, featuring all necessary informations (destination, ID, etc.). When a packet is generated, a Bernoulli process determines its length.

We use OMNET++ to test the bandwidth allocation protocol. We focus on inter-cluster communications as we aim to show the benefits of the dynamic allocation algorithm. Thus, we discard intra-cluster communications. We also assume infinite capacity transmit queues. With state-of-theart on-chip simulators, generating memory traffic by using benchmark applications is not efficient for more than 1000 cores [10]. So we use synthetic models to mimic memory traffic as a first approach.

\subsubsection{Results - Uniform On-Chip Traffic}

One of the most widely used traffic models for on-chip networks is the uniform model, where each node may generate a packet every cycle with a probability $p$. However, as we have 64 cores per cluster and multiple cycles in an OFDM symbol (with $1 \mathrm{GHz}$ processors, one symbol corresponds to $50 \mathrm{cy}-$ cles), there may be multiple packets generated in a symbol. As aggregation of multiple independent Bernouilli processes is a Poisson process, we model the number of packets generated in a cluster on each symbol as a Poisson distribution. We compare the dynamic allocation (EQPS) with the case

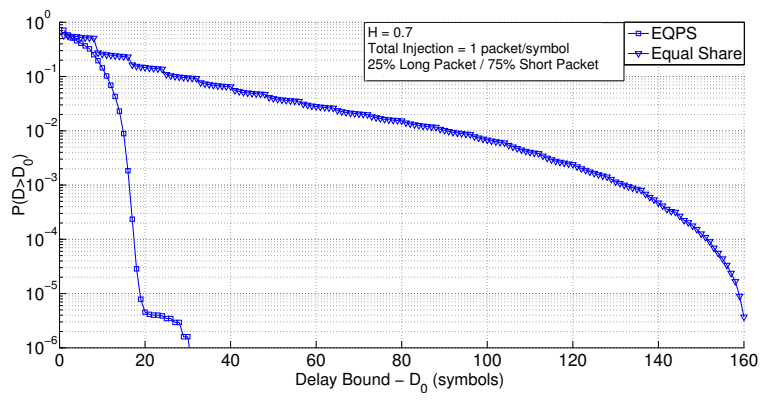

Figure 6: ICDF under realistic traffic model

where subcarriers are statically and equally allocated (Equal Share). Figure 5 shows the packets average latency under Poisson traffic with increasing injection rate. Note that, for injection rates smaller than 0.15 packets/symbol/cluster, Equal Share performs better than EQPS. Indeed, EQPS rearbitrates subcarriers every 8 symbols, based on the instantaneous QSI and a moving average, which does not provide high efficiency when queue length values are small. As traffic load increases and instantaneous queue lengths get bigger and bigger, the need to reallocate bandwidth arises and EQPS becomes more efficient, pushing back the saturation threshold of the network.

\subsubsection{Results - Realistic On-Chip Traffic}

Uniform and Markovian traffic do not accurately capture the features of on-chip traffic. So, new synthetic models have been developed. The fractal and self-similar behavior of CMP memory traffic both in time and space have been observed [1]. One characteristic of memory traffic of shared memory CMP is the long-range dependence (LRD) [17]. LRD reflects the temporal similarity of the traffic generation process in different scales of time intervals. It can be modeled by a power-law decaying covariance function : $\gamma(k)=c k^{-\alpha}$, where $c$ is an arbitrary scalar and $\alpha$ is an exponent reflecting the degree of long-range dependence. One method to characterize and generate stochastic long-range dependent traffic is to use Hurst Parameter, $H$, where $H=(2-\alpha) / 2$ [20]. A synthetic traffic model for cache-coherent CMPs, which attains an 95\% accuracy compared to real application traces has been proposed [17]. We choose this model to test the $\mathrm{RF}$ NoC and EQPS algorithm under realistic on-chip traffic conditions, with $H=0.7$ (an approximate mean for all kind of applications). To generate traffic with Hurst parameter in OMNET++, we use the method explained in [20], where the number of generated packets by a node at any instance is determined by the aggregation of many Pareto distributed $O N-O F F$ processes (we set this number to 500, which is fairly sufficient for each cluster). Similarly, clusters injection rates are assumed to be Gaussian distribution.

We tested our algorithm under non-uniform traffic and plotted the Inverse Cumulative Probability Density Function (ICDF), the probability that the latency of a packet is larger than a delay bound, $P\left(D>D \_0\right)$. Figure 6 shows ICDF for EQPS and Equal Share, with an injection rate of $1 \mathrm{flit} /$ symbol/cluster. It shows a $\times 6$ improvement, showing the benefits of the EQPS algorithm.

\section{CONCLUSION}

Current RF and optical NoC create multiple channels requiring several transceivers per cluster, which limit variety 
and granularity of reconfiguration schemes. We propose in this paper a dynamically reconfigurable RF NoC based on OFDMA. We describe the hierarchical architecture of the CMP using the RF NoC and propose a dynamic bandwidth allocation algorithm. Static analysis shows that RF NoC average latency is comparable with mesh average latency for P2P communications but up to $\times 3$ lower for broadcast. Under realistic traffic models, the dynamic bandwidth allocation algorithm provides up to $\times 6$ lower latency than static schemes. To comfort those promising results, a SystemC model of the architecture is under development so future work can evaluate more precisely performances with benchmarks. Moreover, one can imagine new cache coherency protocols taking advantage of the low cost broadcast of the proposed RF NoC.

\section{ACKNOWLEDGMENTS}

This work has been supported by the French National Research Agency under grant ANR-12-INSE-0004.

\section{REFERENCES}

[1] P. Bogdan and R. Marculescu. Statistical physics approaches for network-on-chip traffic characterization. In IEEE/ACM International Conf on Hardware/Software Codesign, pages 461-470, 2009.

[2] M. Chang, J. Cong, A. Kaplan, M. Naik, G. Reinman, E. Socher, and S. Tam. Cmp network-on-chip overlaid with multi-band rf-interconnect. In High Performance Computer Architecture (HPCA), IEEE International Symposium on, pages 191-202, 2008.

[3] M. Chang, V. P. Roychowdhury, L. Zhang, H. Shin, and Y. Qian. Rf/wireless interconnect for inter-and intra-chip communications. Proceedings of the IEEE, 89(4):456-466, 2001.

[4] S. Deb, K. Chang, M. Cosic, A. Ganguly, P. Pande, D. Heo, and B. Belzer. Cmos compatible many-core noc architectures with multi-channel millimeter-wave wireless links. In Great Lakes Symposium on VLSI (GLSVLSI), pages 165-170. ACM, 2012.

[5] Y. Duan and E. Alon. A 12.8 gs/s time-interleaved adc with $25 \mathrm{ghz}$ effective resolution bandwidth and 4.6 enob. Solid-State Circuits, IEEE Journal of, 49(8):1725-1738, 2014.

[6] C.-S. Gong, C.-T. Hong, K.-W. Yao, and M.-T. Shiue. A low-power area-efficient sram with enhanced read stability in $0.18-\mu \mathrm{m}$ cmos. In Circuits and Systems, APCCAS 2008, IEEE Asia Pacific Conference on, pages 729-732, 2008.

[7] J. Hu, J. Xu, M. Huang, and H. Wu. A 25-gbps 8-ps/mm transmission line based interconnect for on-chip communications in multi-core chips. In IEEE MTT-S International Microwave Symposium Digest (IMS), pages 1-4, 2013.

[8] ITRS. International technology roadmap for semiconductors report, 2013 version.

[9] K. Jongsun, B. Gyungsu, and M. Chang. A low-overhead and low-power rf transceiver for short-distance on-and off-chip interconnects. IEICE Transactions on Electronics, 94(5):854-857, 2011.

[10] G. Kurian, J. Miller, J. Psota, J. Eastep, J. Liu, J. Michel, L. Kimerling, and A. Agarwal. Atac: A 1000-core cache-coherent processor with on-chip optical network. In International Conference on Parallel Architectures and Compilation Techniques, pages 477-488. ACM, 2010.

[11] J. Lee, M. Zhu, K. Choi, J. H. Ahn, and R. Sharma. $3 \mathrm{~d}$ network-on-chip with wireless links through inductive coupling. In International SoC Design Conference (ISOCC), pages 353-356. IEEE, 2011.

[12] F. Li, C. Nicopoulos, T. Richardson, Y. Xie, V. Narayanan, and M. Kandemir. Design and management of $3 \mathrm{~d}$ chip multiprocessors using network-in-memory. ACM SIGARCH Computer Architecture News, 34(2):130-141, 2006.

[13] I. O'Connor. Optical solutions for system-level interconnect. In International Workshop on System level Interconnect Prediction, pages 79-88. ACM, 2004.

[14] Y. Pan, J. Kim, and G. Memik. Tuning nanophotonic on-chip network designs for improving memory trafics. InPICA@MICRO2009.

[15] V. Pavlidis and E. Friedman. 3-d topologies for networks-on-chip. Very Large Scale Integration (VLSI) Systems, IEEE Trans. on, 15(10):1081-1090, 2007.

[16] K. Seong, R. Narasimhan, and J. M. Cioffi. Queue proportional scheduling in gaussian broadcast channels. In IEEE International Conference on Communications (ICC), pages 1647-1652, 2006.

[17] V. Soteriou, H. Wang, and L.-S. Peh. A statistical traffic model for on-chip interconnection networks. In IEEE International Symposium on Modeling, Analysis, and Simulation of Computer and Telecommunication Systems (MASCOTS), pages 104-116, 2006.

[18] S.-N. Tang, J.-W. Tsai, and T.-Y. Chang. A 2.4-gs/s fft processor for ofdm-based wpan applications. Circuits and Systems II: Express Briefs, IEEE Transactions on, 57(6):451-455, 2010.

[19] D. Vantrease, R. Schreiber, M. Monchiero, M. McLaren, N. P. Jouppi, M. Fiorentino, A. Davis, N. Binkert, R. G. Beausoleil, and J. H. Ahn. Corona: System implications of emerging nanophotonic technology. ACM SIGARCH Computer Architecture News, 36(3):153-164, 2008.

[20] W. Willinger, M. S. Taqqu, R. Sherman, and D. V. Wilson. Self-similarity through high-variability: statistical analysis of ethernet lan traffic at the source level. Networking, IEEE/ACM Transactions on, 5(1):71-86, 1997.

[21] C. Xiao, F. Chang, J. Cong, M. Gill, Z. Huang, C. Liu, G. Reinman, and H. Wu. Stream arbitration: Towards efficient bandwidth utilization for emerging on-chip interconnects. ACM Transactions on Architecture and Code Optimization (TACO), 9(4):60, 2013.

[22] Q. Xu, D.-n. Cheng, and Y.-d. Fu. Traffic feature distribution analysis based on exponentially weighted moving average. In Computer Science and Automation Engineering (CSAE), IEEE International Conference on, volume 1, pages 535-539, 2012.

[23] Y. Ye, L. Duan, J. Xu, J. Ouyang, M. Hung, and Y. Xie. 3d optical networks-on-chip (noc) for multiprocessor systems-on-chip (mpsoc). In $3 D$ System Integration (3DIC), IEEE International Conference on, pages 1-6, 2009. 